\title{
Disrupting the Pathways of Social Determinants of Health: Doula Support during Pregnancy and Childbirth
}

\author{
Katy B. Kozhimannil, PhD, MPA, Carrie A. Vogelsang, MPH, \\ Rachel R. Hardeman, PhD, MPH, and Shailendra Prasad, MD, MPH
}

Purpose: The goal of this study was to assess perspectives of racially/ethnically diverse, low-income pregnant women on how doula services (nonmedical maternal support) may influence the outcomes of pregnancy and childbirth.

Methods: We conducted 4 in-depth focus group discussions with low-income pregnant women. We used a selective coding scheme based on 5 themes (agency, personal security, connectedness, respect, and knowledge) identified in the Good Birth framework, and we analyzed salient themes in the context of the Gelberg-Anderson behavioral model and the social determinants of health.

Results: Participants identified the role doulas played in mitigating the effects of social determinants. The 5 themes of the Good Birth framework characterized the means by which nonmedical support from doulas influenced the pathways between social determinants of health and birth outcomes. By addressing health literacy and social support needs, pregnant women noted that doulas affect access to and the quality of health care services received during pregnancy and birth.

Conclusions: Access to doula services for pregnant women who are at risk of poor birth outcomes may help to disrupt the pervasive influence of social determinants as predisposing factors for health during pregnancy and childbirth. (J Am Board Fam Med 2016;29:308-317.)

Keywords: Birth; Cultural Diversity; Health Care Disparities; Populations, Underserved; Social Determinants of Health

Social determinants of health (SDOHs), including economic stability, level of education, neighborhood and environment, and social relationships and

This article was externally peer reviewed.

Submitted 12 September 2015; revised 3 November 2015; accepted 19 November 2015.

From the Division of Health Policy and Management, University of Minnesota School of Public Health, Minneapolis (KBK, CAV); the Division of Health Care Policy \& Research, Mayo Clinic College of Medicine, Rochester, MN (RRH); and the Department of Family Medicine and Community Health, University of Minnesota Medical School, Minneapolis (SP).

Funding: Research reported in this article was supported a Community Health Collaborative Grant from the National Center for Advancing Translational Sciences of the National Institutes of Health (award no. UL1TR000114).

Conflict of interest: none declared.

Disclaimer: The content of this article is solely the responsibility of the authors and does not necessarily represent the official views of the National Institutes of Health.

Corresponding author: Katy B. Kozhimannil, PhD, MPA, Division of Health Policy \& Management, University of Minnesota School of Public Health, 420 Delaware St SE, D359 Mayo, MMC 729, Minneapolis, MN 55455 (E-mail: kbk@umn.edu). interactions, ${ }^{1}$ are predisposing factors that influence health outcomes. ${ }^{2-4}$ The impact of SDOHs is heightened among vulnerable populations, and they play a crucial role in maternal and infant health outcomes. ${ }^{5}$

For example, women who have or develop conditions such as diabetes or hypertension during pregnancy are more likely to have a primary cesarean delivery or preterm birth. ${ }^{6}$ Development of these and related conditions, including obesity, relates to the structural and environmental factors that affect access to exercise and nutrition. ${ }^{6}$ Women who experience intimate partner violence and exposure to abuse are more likely to have little or no prenatal care, be hospitalized during pregnancy, and give birth to low-birthweight infants. ${ }^{7,8}$ Unsafe neighborhoods and adverse environmental exposures increase the likelihood of preterm birth. ${ }^{9-11}$ Low health literacy among pregnant women is associated with low attendance of prenatal care visits and poor birth out- 
Figure 1. Conceptual model of the pathways between social determinants of health and birth outcomes and the role of non-medical support.

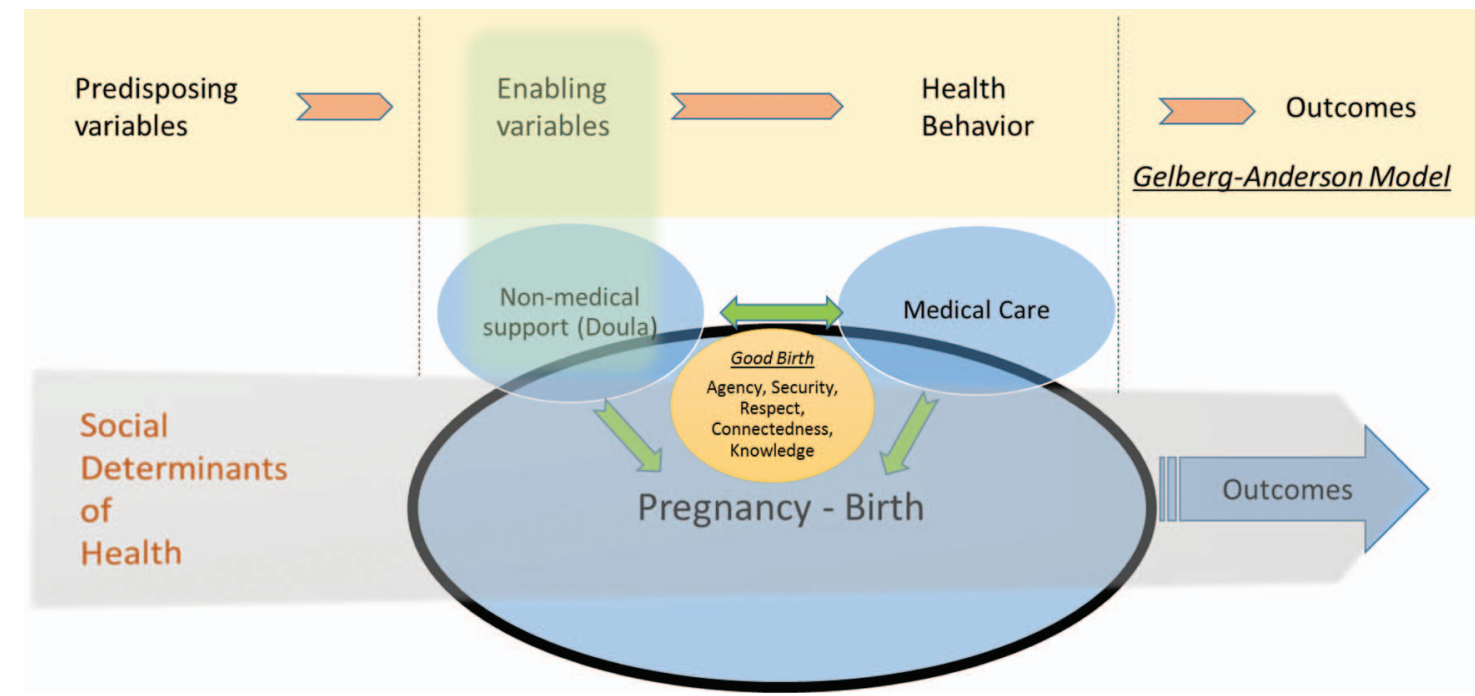

comes. ${ }^{12,13}$ Women with low socioeconomic status have greater chances of having a low-birth-weight infant or a preterm birth. ${ }^{14}$ In addition, pregnant women with limited social support are more likely to have a low-birth-weight infant. ${ }^{15}$

The pathways between SDOHs and birth outcomes have contributed to pervasive racial/ethnic disparities in maternal health and health care. ${ }^{16-18}$ Longstanding and complex sociodemographic and historic factors perpetuate the challenges women of color face in achieving positive birth outcomes. ${ }^{19-20}$ These disparities have persisted despite clinical and nonclinical approaches and interventions in the health care setting, and few solutions with the potential to effectively disrupt the pathway between SDOHs and poor birth outcomes have been identified. ${ }^{16,21}$

Nonmedical interventions are preferred options in addressing SDOHs. ${ }^{22}$ Doulas are trained professionals who provide continuous, 1-on-1 emotional and informational support during the perinatal period. Similar to community health workers, they are not medical professionals and do not provide medical services, but work alongside health care providers. Studies show that doula care is associated with lower epidural use and cesarean delivery rates, shorter labors, higher rates of spontaneous vaginal birth, and higher levels of satisfaction. ${ }^{23-27}$ Low-income women and women of color, who have the highest risk of poor birth outcomes, are also the most likely groups to report wanting, but not having, access to doula services. ${ }^{26}$ The current evidence base is lacking effective means of mitigating the effects of SDOHs on birth outcomes for these high-risk populations. The goal of this study was to assess perspectives among racially/ethnically diverse, low-income pregnant women of how access to and support from a doula may influence the outcomes of pregnancy and childbirth.

\section{Methods}

\section{Conceptual Model}

We used the Gelberg-Anderson model of health behavior $^{4}$, and components of the Good Birth framework ${ }^{28}$ to create a conceptual model to describe the role of doulas-and of medical care-in the pathway between SDOHs and birth outcomes. SDOHs are present before, during, and after pregnancy, but their effects on birth outcomes may be moderated by the quality of clinical care and the nonmedical support a woman receives (Figure 1).

The Gelberg-Anderson model of health behavior focuses on vulnerable populations. ${ }^{4}$ The Predisposing, Enabling, and Need components of this model predict personal health practices, including the use of health services. The Predisposing domain includes "demographic characteristics" and "social structure patterned social arrangements in society that are both emergent from and determinant of the actions of the individuals." 4 Social structure includes a variety of components that create the context by which SDOHs are manifested and maintained. Thus our study considers the 
$\mathrm{SDOHs}^{1}$ to be a component of the Predisposing domain. The Predisposing variables affect Enabling variables (eg, social support, health services resources, ability to negotiate the system), which subsequently affect Need. In our framework the Enabling variables include doula services, which are directly associated with a pregnant woman's perceived need for help. Gelberg and colleagues ${ }^{4}$ suggest that health behaviors and health outcomes will subsequently be impacted by Need. When placing this model within the context of childbirth within vulnerable populations, we also consider the elements of Agency, Personal Security, Connectedness, Respect, and Knowledge that Dr. Anne Lyerly identified as characteristic of a good birth. ${ }^{28}$ These elements of a good birth can inform strategies to meet the physical and emotional needs of women during pregnancy and childbirth. ${ }^{28}$ Our model frames the context in which the trajectory of a woman's pregnancy and the SDOHs may be influenced by the support of a doula, the clinical care she receives, and potentially by the interactions between the doula and the patient's clinician.

\section{Study Participants}

Thirteen racially/ethnically diverse, low-income pregnant women participated in 4 focus group discussions that were held at 3 locations in Minneapolis, Minnesota, in November and December 2014. Multiple methods (flyers, E-mails, and word of mouth) were used for recruitment. Inclusion criteria included pregnancy and fluency in English. The role of a doula was explained at the outset of the interview, and prior experience with a doula was not required so as not to exclude potential participants who may not have been able to afford or access doula services. All participants consented to participate using a human subjects protection process approved by the University of Minnesota institutional review board (code no. 1403S49085).

\section{Data and Measurement}

In collaboration with community-based partners, we developed and pilot-tested a questionnaire to guide semistructured focus group discussions. These discussions were facilitated by 2 investigators (RRH and CAV), both of whom are trained and experienced in qualitative data collection and analysis. Each focus group included between 2 and 6 participants. We had planned for 3 focus groups of 5 to 7 women; however, inclement weather pre- cluded participation for several women in the first scheduled focus group, so we scheduled a fourth focus group meeting to allow their participation. Data saturation was achieved with 4 focus groups. All of the focus group discussions were recorded and transcribed using CaptionSync Professional Transcription, a service provided by Automatic Sync Technologies, LLC (Seattle, WA). Manual notes taken by the facilitators (RRH, CAV) were used to augment the transcripts where comments were inaudible during the recording.

Questions focused on reasons for and barriers to doula support, and the ways doulas influence pregnancy and birth, based on prior research. ${ }^{24-27,29}$ We used the themes of the good birth framework (agency, personal security, respect, knowledge, connectedness) $)^{28}$ in a deductive approach to code the transcripts. We also created and used separate codes that highlighted (1) the mechanisms associated with doula support and healthy pregnancy, and (2) the relationship of these mechanisms with SDOHs.

\section{Analysis}

The initial coding was separately and independently validated using a coanalysis method among the authors (CV, RRH, KBK). ${ }^{30}$ Coding was conducted in a shared Microsoft Excel document (Microsoft Corp., Redmond, WA). After the first round of coding, we met to discuss differences among coders and to refine codes and definitions for clarity. Then, one of the authors (CAV) led a second round of coding, grouping each of the codes to identify which themes emerged as patterns across the focus groups. We then followed the same 2-step process to code the transcripts for specific mechanisms of doula support that were associated with birth outcomes.

\section{Results}

The study participants represented a racial/ethnically diverse group of women, as described in Table 1. Participants were nearly evenly split between nulliparous and parous, and three quarters of participants had a doula supporting them during their current pregnancy. Nearly $40 \%$ of women who participated in the focus groups voluntarily disclosed that their pregnancy was complicated by a medical condition (such as hypertension, prior preterm birth, or gestational diabetes). 
Table 1. Descriptive Characteristics of Focus Group Participants

\begin{tabular}{lc}
\hline Characteristics & $\begin{array}{c}\text { Participants, } \\
\mathrm{n}(\%)\end{array}$ \\
\hline Race/ethnicity & \\
African American & $5(38.5)$ \\
African & $4(30.7)$ \\
Native American & $2(15.4)$ \\
White & $2(15.4)$ \\
Parity & \\
First pregnancy (nulliparous) & $6(46.2)$ \\
Experienced mother (parous) & $7(53.8)$ \\
Doula with current pregnancy & \\
Yes & $10(76.9)$ \\
No & $3(23.1)$ \\
Voluntarily disclosed high-risk medical status & \\
Yes & $5(38.5)$ \\
No & $8(61.5)$ \\
\hline
\end{tabular}

Table 2 contains information on each of the key themes, with illustrative quotes, as described below.

\begin{abstract}
Agency
"[Having a doula] helps prepare you mentally; like it is gotten me more in the mind-set of . . . the confidence throughout the pregnancy knowing that I can do this. ..."

Agency is the capacity of an individual to act or to make his/her own choices (as opposed to being someone to whom things happen). ${ }^{28}$ Low-income and racially/ethnically diverse women suffer a lack of agency in their medical care. ${ }^{31,32}$ Our findings suggest that doulas play an important role in equipping low-income, diverse pregnant women with agency by either prompting the expression of concerns or by facilitating interactions with the health care provider (Table 2, quote 2). Having a doula plays an important role in a woman's ability to make an informed decision while positively influencing her belief in herself (Table 2 quote 1).
\end{abstract}

\section{Personal Security}

"... I talk to the doctor ... and I am calling the doula right after that. . . . Like, I am scared. . . and she's like, oh, no do not be. ... It is very comforting to know that you have somebody [who] has your back."

Physical and emotional safety plays an important role in pregnancy and childbirth. Feeling secure, comfortable, and calm is particularly crucial for women contending with complex social circumstances (eg, an unstable living situation, an unsupportive partner). As reflected in the above quote, the respondent's doula contributed to her personal security by addressing her health concerns after an encounter with her provider. This concept of security extends to the incorporation of culturally concordant beliefs about childbirth and personal safety (Table 2, quote 5).

\section{Respect}

“... Having someone that is not only knowledgeable, but can put things in layman's terms, in a way that you understand it and respects your culture ... your well-being, your upbringing and things about you ... to make sure that the baby is okay too."

Respect is critical to a patient-centered experience, and a physician's respect of a patient's autonomy is often cited as an important goal of the birthing process. ${ }^{28}$ Further, respect is the basis of informed consent. ${ }^{28}$ Autonomy in decision making is a marker of respect and was discussed among focus group participants as a key component (Table 2 , quote 8 ). This theme was echoed throughout the groups, and there was consensus that a doula's presence, particularly during the childbirth process, would facilitate greater autonomy and respect in decision making.

\section{Knowledge}

"My reasons for wanting a doula. [It is] because I do not have nobody right now, and if I go into labor, ... I do not know the techniques or how to calm down."

Our findings suggest that doulas play a critical role in imparting knowledge to their clients and empowering them to become knowledgeable about the physiologic process of pregnancy. Some women gain this knowledge from their health care providers; however, many of the participants suggested that they often did not fully understand some of the things their provider shared with them. In these instances they relied on their doula to help "translate" their clinical encounters. In addition, having a doula present to share techniques and pass on wisdom and birth strategies is important (Table 2, quote 12). Doulas also play an important role in connecting women with resources to gain new knowledge as they prepare for childbirth (Table 2, quotes 11 and 13). 


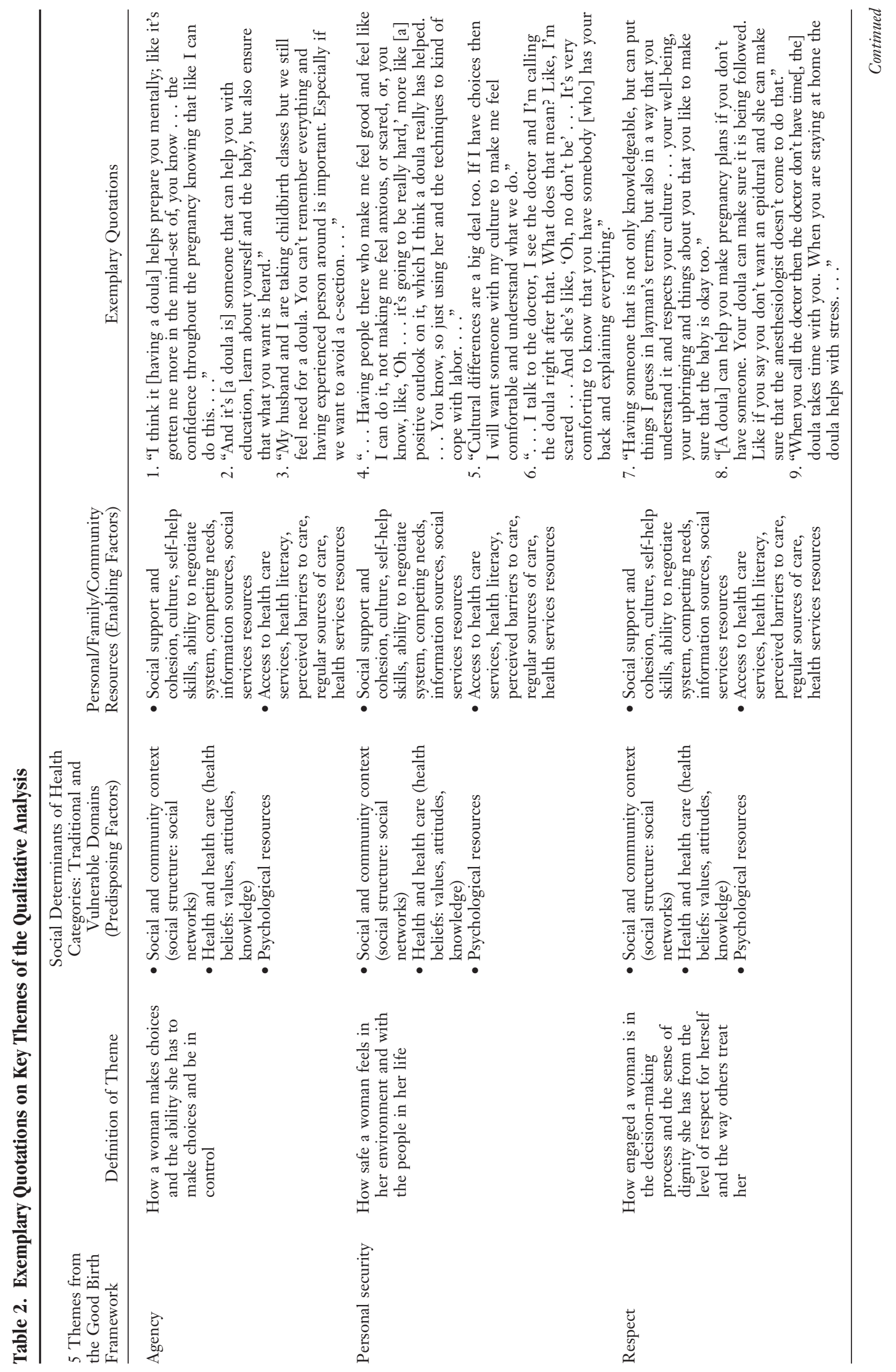




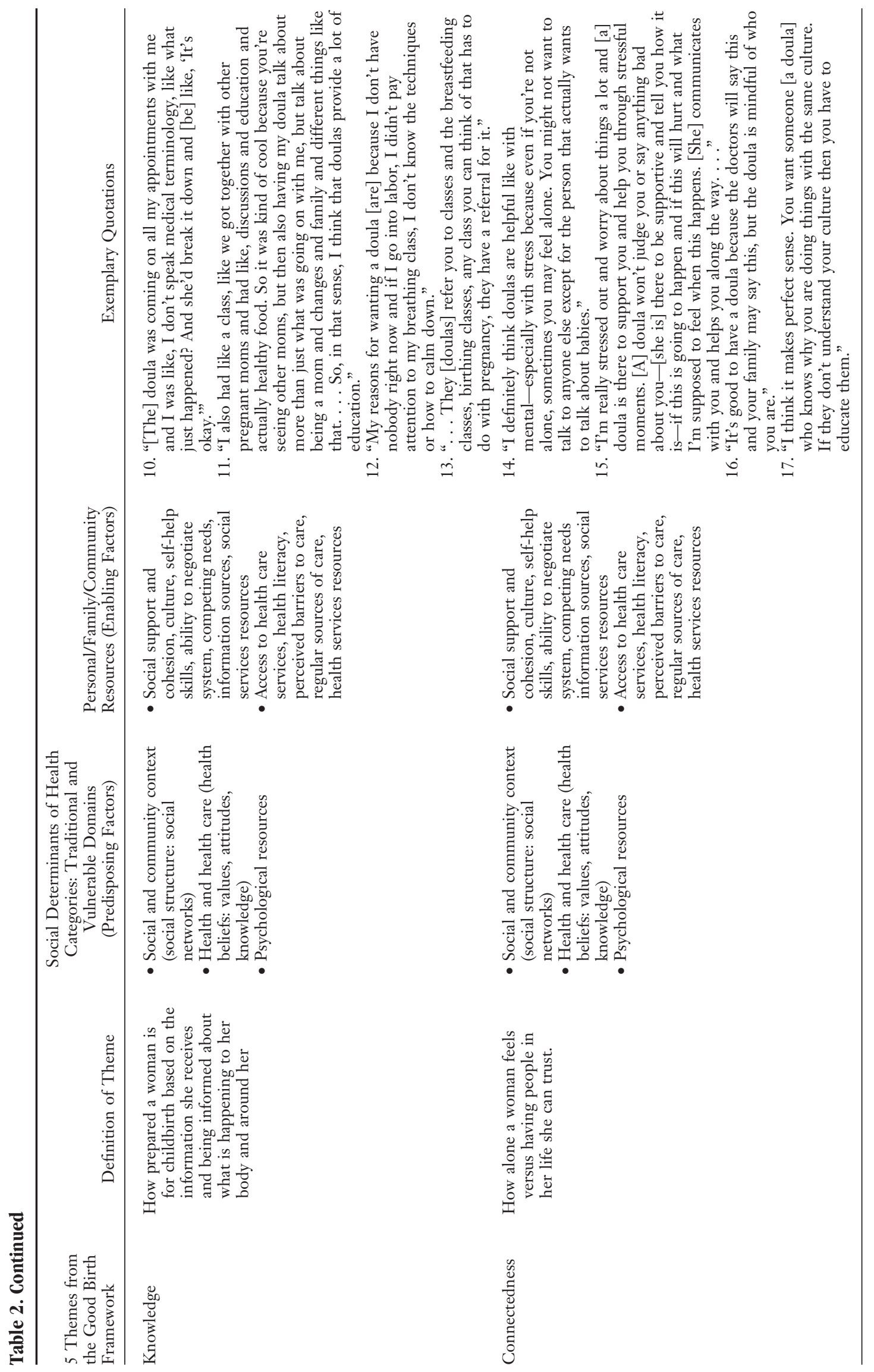




\section{Connectedness}

"... It is good to have a doula because the doctors will say this and your family may say this, but the doula is mindful of who you are."

Connectedness considers the level at which a woman feels connected to the resources that are available, her clinicians, her infant, and the support people in her life-including her doula. Participants observed that doulas play an important role in ensuring that women who lack social support do not feel isolated (Table 2, quote 14). Women expressed that the connection with their doula would make a difference in their pregnancy and childbirth, sometimes even more so than a health care provider or family member (Table 2, quote 16). Women found the connection with their doula to be important for the general support that the doula provides, beyond specific knowledge or guidance in the birth process (Table 2, quote 15). Many of the participants described stressful life situations and emphasized the desire to connect with a person who shared their culture and background (Table 2, quote 17).

\section{Discussion}

Participant responses revealed that nonmedical support from a doula could play a role in helping women overcome barriers to achieving a healthy pregnancy and childbirth. Women's responses aligned with 2 key categories of SDOHs defined in HealthyPeople $2020^{1}$ : health and health care; and social and community context. While the skills they bring and the support they provide are nonmedical, doulas play a role in pregnant women's ability to access health services and in the quality of care they receive by addressing the women's health literacy and social support needs, as well as through interaction with prenatal and intrapartum care providers. While clinicians provide direct patient care in the context of the health and health care SDOH category, study participants also identified doulas as facilitators of improved patient-provider interactions that influence satisfaction with the birth experience and favorable birth outcomes.

Much of the current research on successful interventions to address SDOHs at the time of childbirth come from the international context, ${ }^{33}$ but programs addressing SDOHs for maternal and child health are increasingly being adopted in the United States, largely owing to the persistence of disparities despite medically focused interventions. For example, a community-based project in California serves as a model for successfully addressing SDOHs to reduce racial disparities and improve birth outcomes for African American women. ${ }^{34}$ This program shifted prenatal care and case management to include support groups that educate, inform, empower, and connect women socially, culturally, and financially. ${ }^{34}$ Our findings are consistent with the those that emerged from the California initiative. However, our findings extend the learnings gleaned from individual projects and programs to explore a concept (nonmedical support) that can be integrated within health care financing and delivery systems to affect system change and potentially create long-term, sustainable solutions to persistent disparities in birth outcomes.

Much prior research on doula care has been conducted among white, upper-middle-class women and/or in a randomized controlled trial context. $^{23,29}$ While emerging research shows that the known benefits of doula care may be even greater among vulnerable populations, ${ }^{24,26}$ those who could most benefit from doula care frequently have the least access to it. ${ }^{23}$ Future work should examine the perspectives of doulas and of clinicians to further inform the conceptual model developed here. In addition, policy and clinical efforts to increase access to doula services should address cultural, financial, and geographic barriers to care identified by pregnant women.

\section{Implications for Policy}

Access to culturally concordant care and support during childbirth was noted as a potential benefit of doula services by the women in our study, but a lack of diversity among the doula workforce was seen as a potential barrier. Difficulty in ensuring representativeness among doulas is likely exacerbated by the fact that doula services are rarely covered by health insurance, thus creating a barrier to entry into this profession that disproportionately affects low-income communities. ${ }^{29,35}$ Recent research on doula care and cost savings, especially among low-income women, has ignited discussion regarding reimbursement of doula care by health insurance programs, including Medicaid. ${ }^{24,26,27}$ Two states (Oregon and Minnesota) currently allow Medicaid reimbursement for doula services. Minnesota passed legislation in May 2013 establishing Medicaid reimbursement for doulas, which became effec- 
tive starting September 25, 2014, upon federal approval. $^{36}$ Implementation challenges have been substantial and include alack of awareness about doula services on the part of pregnant women, maternity care clinicians, hospitals, and clinics, and the health insurance plans that provide coverage to Medicaid beneficiaries. ${ }^{37}$ This research provides a framework for understanding how doula care may influence the pathways between SDOHs and birth outcome, which may inform future efforts to expand health insurance coverage of doula services and integrate nonmedical support within health care delivery systems.

\section{Implications for Clinical Practice}

Means of addressing SDOHs are not inherently present in current health care delivery models. In childbirth in particular there is a tendency toward a "technocratic" approach that privileges medical care over nonmedical support. ${ }^{38}$ Pregnancy and childbirth are critical junctures in the life course, when the impacts of social determinants are heightened. Increasingly, women giving birth in the United States are doing so in isolation, with a lack of personal, social, and emotional support. ${ }^{39,40} \mathrm{Re}-$ cent studies have highlighted the importance of trust within the patient-provider relationship, the challenges this presents for low-income women, and the resulting effects on overall quality and disparities in maternal and child health outcomes. ${ }^{41,42}$

Prior research has suggested the need for adequate clinical care, as well as personal support at the individual level, during pregnancy. ${ }^{41}$ Doulas were seen in our study as providing social support to help improve communication between low-income, racially/ethnically diverse pregnant women and their health care providers via an increase in women's agency and knowledge during pregnancy. Women in our study indicated that doulas helped to create an environment of trust. Our study reflected the sense of engagement and connectedness participants felt in the presence of doulas, and noted that this presence can enhance the clinical encounter via improving the process of informed consent and increasing patient satisfaction. ${ }^{23}$

\section{Limitations}

The sample used for this study included 13 women and was a convenience sample from 1 metropolitan area of the United States; thus broad generalizations cannot be made. This exploratory study helped to generate a conceptual model that sets forth hypotheses for future work but does not establish a causal pathway. The focus groups took place during the early phases of implementation of Medicaid coverage of doula services in Minnesota and do not reflect full implementation of that policy, which may influence access to doula care by vulnerable populations. These results indicate the need for further investigation of the role of nonmedical support in addressing SDOHs.

\section{Conclusions}

Improving access to doula services by pregnant women who are at risk of poor birth outcomes may enhance clinical efforts to overcome the pervasive influence of SDOHs on pregnancy and childbirth. This study contributes to the growing body of evidence that doulas are a social support intervention that can influence the pathways between social determinants and birth outcomes by addressing some of the underlying issues that evade clinical approaches to persistent disparities.

The authors gratefully acknowledge data entry support from Shruthi Kamisetty and input and feedback provided by Amanda Huber, CMN; Rita O'Reilly, CNM; Debby Prudhomme; and Mary Williams, LPN. This research would not have been possible without the collaboration of our community partners, Everyday Miracles, Cultural Wellness Center, and Missionaries of Charity, all located in Minneapolis, MN.

\section{References}

1. HealthyPeople 2020. Social determinants of health. Washington, DC: U.S. Department of Health and Human Services, Office of Disease Prevention and Health Promotion. Available from: http:// www.healthypeople.gov/2020/topics-objectives/topic/ social-determinants-health. Accessed July 16, 2015.

2. Babitsch B, Gohl D, von Lengerke T. Re-revisiting Andersen's Behavioral Model of Health Services Use: a systematic review of studies from 1998-2011. Psychosoc Med 2012;9:Doc11.

3. Andersen RM. Revisiting the behavioral model and access to medical care: does it matter? J Health Soc Behav 1995;36:1-10.

4. Gelberg L, Andersen RM, Leake BD. The Behavioral Model for Vulnerable Populations: application to medical care use and outcomes for homeless people. Health Serv Res 2000;34:1273-302.

5. Halfon L, Larson K, Lu M, Tullis E, Russ S. Lifecourse health development: past, present and future. Child Health J 2014;18:344-65. 
6. Rosenberg TJ, Garbers S, Lipkind H, Chiasson MA. Maternal obesity and diabetes as risk factors for adverse pregnancy outcomes: differences among 4 racial/ethnic groups. Am J Public Health 2005;95:1545-51.

7. Parker B, McFarlane J, Soeken K. Abuse during pregnancy: effects on maternal complications and birth weight in adult and teenage women. Obstet Gynecol 1994;84.3:323-8.

8. Cokkinides VE, Coker AL, Sanderson M, Addy C, Bethea L. Physical violence during pregnancy: maternal complications and birth outcomes. Obstet Gynecol 1999;93(5 Pt 1):661-6.

9. Farley TA, Mason K, Rice J, Habel JD, Scribner R, Cohen DA. The relationship between the neighbourhood environment and adverse birth outcomes. Paediatr Perinat Epidemiol 2006;20:188-200.

10. Stillerman KP, Mattison DR, Guidice LC, Woodruff TJ. Environmental exposures and adverse pregnancy outcomes: a review of the science. Reprod Sci 2008;15.7:631-50.

11. Kruger DJ, Munsell MA, French-Turner T. Using a life history framework to understand the relationship between neighborhood structural deterioration and adverse birth outcomes. J Soc Evolut Cult Psychol 2011;5:260-74.

12. Bennett IM, Switzer J, Aguirre A, Evans K, Barg F. "Breaking it down": patient-clinician communication and prenatal care among African women of low and higher literacy. Ann Fam Med 2006;4: 334-40.

13. Endres LK, Sharp LK, Haney E, Dooley SL. Health literacy and pregnancy preparedness in pregestational diabetes. Diabetes Care 2004;27:331-4.

14. Luo Z-C, Wilkins R, Kramer MS; Fetal and Infant Health Study Group of the Canadian Perinatal Surveillance System. Effect of neighbourhood income and maternal education on birth outcomes: a population-based study. CMAJ 2006;174:1415-20.

15. Feldman PJ, Dunkel-Schetter C, Sandman CA, Wadhwa PD. Maternal social support predicts birth weight and fetal growth in human pregnancy. Psychosom Med 2000;62:715-25.

16. Lu MC, Halfon N. Racial and ethnic disparities in birth outcomes: a life-course perspective. Matern Child Health J 2003;7:13-30.

17. ACOG Committee on Health Care for Underserved Women. ACOG committee opinion. Number 317, October 2005. Racial and ethnic disparities in women's health. Obstet Gynecol 2005;106:889-92.

18. ACOG committee opinion no. 586: health disparities in rural women. Obstet Gynecol 2014;123(2 Pt 1):384-8.

19. Giscombé CL, Lobel M. Explaining disproportionately high rates of adverse birth outcomes among African Americans: the impact of stress, racism, and related factors in pregnancy. Psychol Bull 2005;131:662-83.

20. Hogan VK, Shanahan ME, Rowley DL. Current approaches to reducing premature births and impli- cations for disparity elimination. In: Handler A, Kennelly J, Peacock N, eds. Reducing racial/ethnic disparities in reproductive and perinatal outcomes: the evidence from population-based interventions. New York, NY: Springer; 2011:181-207.

21. Livingood WC, Brady C, Pierce K, Atrash H, Hou T, Bryant T III. Impact of pre-conception health care: evaluation of a social determinants focused intervention. Matern Child Health J 2010;14:382-91.

22. Braveman P, Egerter S, Williams DR. The social determinants of health: coming of age. Annu Rev Public Health 2011;32:381-98.

23. Hodnett E, Gates S, Hofmeyr G, Sakala C. Continuous support for women during childbirth (review). Cochrane Database Syst Rev 2013;7:CD003766.

24. Kozhimannil KB, Hardeman RR, Attanasio LB, Blauer-Peterson C, O'Brien M. Doula care, birth outcomes, and costs among Medicaid beneficiaries. Am J Public Health 2013;103:e113-21.

25. Kozhimannil KB, Attanasio LB, Hardeman RR, O'Brien M. Doula care supports near-universal breastfeeding initiation among diverse, low-income women. J Midwifery Womens Health 2013;58:37882.

26. Kozhimannil KB. Attanasio LB, Jou J, Joarnt LK, Johnson PJ, Gjerdingen DK. Potential benefits of increased access to doula support during childbirth. Am J Manag Care 2014;20:e340-52.

27. Kozhimannil KB, Hardeman RR, Alarid-Escudero F, Vogelsang CA, Blauer-Peterson C, Howell EA. Modeling the cost-effectiveness of doula care associated with reductions in preterm birth and cesarean delivery. Birth 2016;43:20-7.

28. Lyerly AD. A good birth: finding the positive and profound in your childbirth experience. Penguin; 2013.

29. Lantz PM, Low LK, Varkey S, Watson RL. Doulas as childbirth paraprofessionals: results from a national survey. Womens Health Issues 2005;15:109-16.

30. Creswell JW, Maietta RC. Systematic procedures of inquiry and computer data analysis software for qualitative research. Handbook of Research Design and Social Measurement. Thousand Oaks, CA: Sage Publications; 2001;6:143-84.

31. O'Malley AS, Sheppard VB, Schwartz M, Mandelblatt $\mathrm{J}$. The role of trust in use of preventive services among low-income African-American women. Prev Med 2004;38:777-85.

32. Sword W. A socio-ecological approach to understanding barriers to prenatal care for women of lowincome. J Adv Nurs 1999;29:1170-7.

33. United Nations Development Program, Bureau of Development Policy. Discussion paper: a social determinants approach to maternal health. Roles for development actors. October 19, 2011. Available from: http://www.undp.org/content/dam/undp/ library/Democratic\%20Governance/Discussion\%20 
Paper\%20MaternalHealth.pdf. Accessed August 10, 2015.

34. California Black Infant Health Program. Available from: http://www.cdph.ca.gov/programs/BIH/Pages/ default.aspx. Accessed March 25, 2016

35. Morton CH, Basile M. Medicaid coverage for doula care: re-examining the arguments through a reproductive justice lens, part one. Science \& Sensibility, March 28, 2013. Available from: http://www.scienceandsensibility. org/?p=6461. Accessed March 31, 2014.

36. Doula services medical assistance (MA) coverage requirement. Minnesota Senate bill 699, section 256B.0625, subdivision 28b. Available from: https:// legiscan.com/MN/text/SF699/id/752534. Accessed June 29, 2015.

37. Kozhimannil KB, Almanza J, Vogelsang CA, Hardeman RR. Medicaid coverage of doula services in Minnesota: findings from the first year. University of
Minnesota. December 2015. Available at http://sph .umn.edu/faculty1/hpm/name/katy-kozhimannil/.

38. Davis-Floyd R. The technocratic, humanistic, and holistic paradigms of childbirth. Int J Gynecol Obstet 2001;75:S5-23.

39. Leavitt JW. The growth of medical authority: technology and morals in turn-of-the-century obstetrics. Med Anthropol Q 1987;1:230-55.

40. Leavitt JW. Women and health in America: historical readings. Madison: University of Wisconsin Press; 1999.

41. Aved BM, Irwin MM, Cummings LS, Findeisen N. Barriers to prenatal care for low-income women. West J Med 1993;158:493-8.

42. Sheppard VB, Zambrana RE, O'Malley AS. Providing health care to low-income women: a matter of trust. Fam Pract 2004;21:484-91. 